\title{
Repurposing non-cancer Drugs in Oncology - How many drugs are out there?
}

Pan Pantziarka ${ }^{1,2}$, Vidula Sukhtame $^{3}$, Lydie Meheus ${ }^{1}$, Vikas Sukhatme ${ }^{3,4}$, Gauthier Bouche $^{1}$ (Final list to be discussed)

1 Anticancer Fund, Brussels, 1853 Strombeek-Bever, Belgium

2 The George Pantziarka TP53 Trust, London, UK

3 GlobalCures, Inc, Newton MA 02459, USA

4 Beth Israel Deaconess Medical Center and Harvard Medical School, Boston, MA 02215, USA.

This is a preliminary version that has not been submitted for publication. Changes to the manuscript are currently being done by the authors. Authors' list may change.

\section{Abstract}

\section{Background}

Drug repurposing can speed up access to new therapeutic options for cancer patients. With more than 2000 drugs approved worldwide and 6 relevant targets per drug on average, the potential is quantitatively important. In this paper, we have attempted to quantify the number of non-cancer drugs supported by either preclinical or clinical cancer data.

Methods

A PubMed search was performed to identify non-cancer drugs which could be repurposed in one or more cancer types. Drugs needed at least one peer-reviewed article showing an anticancer effect in vitro, in vivo or in humans.

Results

A total of 235 eligible non-cancer drugs were identified (Table 1). Main charateirstics of the drugs are summarized in Table 2. 67 (29\%) are on the WHO list of essential medicines and 176 (75\%) are offpatent. 133 (57\%) had human data in cancer patient(s). Four were listed in clinical guidelines, namely thalidomide, all-trans retinoic acid, zoledronic acid and non-steroidal anti-inflammatory drugs (NSAID). Several drugs have shown a survival benefit in randomized trials such as cimetidine (colorectal cancer), progesterone (breast cancer) or itraconazole (lung cancer). Several other drugs induced responses in rare tumours, like clarithromycin, timolol or propranolol.

\section{Conclusion}

We have found that the number of off-patent repurposing opportunities is large and increasing. Joint non-commercial clinical development (academics, governments, charities) may bring new therapeutic options to patients at low cost, especially in indications for which the industry has no incentive to invest in. 


\section{Introduction}

Drug repurposing can speed up access to new therapeutic options for cancer patients. Whereas it is not unusual to attempt to find new cancer uses for existing anticancer drugs, less attention and efforts are made to find anticancer uses of non-cancer drugs ${ }^{1}$. However, many non-cancer drugs could be potentially repurposed against cancer though to our knowledge no figures have been put forward so far ${ }^{2}$. One advantage of non-cancer drugs is that they represent a way to adapt to new knowledge about cancer. For instance, Tadalafil (PDE-5 inhibitor, erectile dysfunction), inhibits myeloid-derived suppressor cells (MDSC) in cancer patients ${ }^{3}$. Or propranolol (beta-blocker, hypertension) reduces proliferation and migration of angiosarcoma models ${ }^{4,5}$, by blocking beta-adrenergic receptors expressed by angiosarcoma cells ${ }^{6}$.

With more than 2000 drugs approved worldwide and 6 relevant targets per drug on average ${ }^{7}$, the potential is quantitatively important. What's more, with regular new drug approvals, the toolbox that repurposed drugs represent is growing every year. Even antibodies are now being repurposed (e.g. rituximab in pemphigus ${ }^{8}$ ), sometimes based on the discovery of off-target effects ${ }^{9,10}$.

When a drug loses its patent protection, the incentives for the market authorization holder are also lost. Sometimes, other private entities attempt to protect the new therapeutic use by various means and undertake a commercial development ${ }^{11}$. However, this strategy remains financially risky, which makes it less attractive to investors and venture capitalists compared to other biotech investments. We have called these drugs "financial orphan drugs" ${ }^{12}$.

In this paper, we have attempted to quantify the number of non-cancer drugs supported by either preclinical or clinical cancer data.

\section{Methods}

A PubMed search was performed to identify non-cancer drugs which could be repurposed in one or more cancer types. Drugs needed at least one peer-reviewed article showing an anticancer effect in vitro, in vivo or in humans.

\section{Results}

A total of 235 eligible non-cancer drugs were identified (Table 1). Main characteristics of the drugs are summarized in Table 2. 67 (29\%) are on the WHO list of essential medicines and 176 (75\%) are off-patent. $133(57 \%)$ had human data in cancer patient(s). Four were listed in clinical guidelines, namely thalidomide, all-trans retinoic acid, zoledronic acid and non-steroidal anti-inflammatory drugs (NSAID). In the first 3 cases, pharmaceutical companies took the lead and re-branded or re-formulated the drugs. This was not the case for NSAIDs, listed in desmoid tumours guidelines and used off-label.

Several drugs have shown a survival benefit in randomized trials such as cimetidine (colorectal cancer) ${ }^{13}$, progesterone (breast cancer) ${ }^{14}$ or itraconazole (lung cancer) ${ }^{15}$. Of note, several other drugs induced responses in rare tumours, like clarithromycin ${ }^{16}$, timolol ${ }^{17,18}$ or propranolol ${ }^{19-22}$.

Table 1: List of non-cancer drugs with at least one peer-reviewed paper supporting its use against cancer. 


\begin{tabular}{|c|c|c|}
\hline Drug & Pharmacological class & Main Indications \\
\hline Acetazolamide & $\begin{array}{l}\text { Carbonic anhydrase inhibitors - ATC } \\
\text { Code: S01EC01 }\end{array}$ & Glaucoma, diuretic, epilepsy \\
\hline $\begin{array}{l}\text { Acetaminophen } \\
\text { (paracetamol) }\end{array}$ & NA & Analgesic \\
\hline Agomelatine & Melatonergic antidepressant & Insomnia \\
\hline Albendazole & NA & Anthelmintic \\
\hline Aliskiren & Direct renin inhibitor & Essential hypertension \\
\hline Allopurinol & Xanthine oxidase inhibitors & Gout \\
\hline $\begin{array}{l}\text { All-trans retinoic } \\
\text { acid (tretinoin) }\end{array}$ & NA & Acne, APL \\
\hline Alpha-Lipoic Acid & Thioctic acid - A16AX01 & Diabetic neuropathy (Germany) \\
\hline Amantadine & Anticholinergic & Parkinson, Influenza A \\
\hline Amiloride & Potassium-sparing diuretic & $\begin{array}{l}\text { In congestive heart failure or } \\
\text { hypertension treated with thiazides, to } \\
\text { conserve potassium }\end{array}$ \\
\hline Amiodarone & Antiarrhythmic & Ventricular tachycardia/fibrillation \\
\hline Amitriptyline & Tricyclic Anti-depressant & Depression \\
\hline Amlodipine & Calcium Channel Blocker & Hypertension \\
\hline Amodiaquine & Anti-malaria & Malaria \\
\hline Anakinra & IL-1R antagonist & RA, NOMID, CAPS \\
\hline Anagrelide & $\begin{array}{l}\text { PDE3 inhibitor (Platelet-reducing } \\
\text { agent) }\end{array}$ & Essential thrombocythemia \\
\hline Aprepitant & $\begin{array}{l}\text { Substance } \mathrm{P} / \text { neurokinin } 1 \text { (NK1) } \\
\text { receptor antagonist }\end{array}$ & Nausea, vomiting \\
\hline Aprotinin & $\begin{array}{l}\text { Bovine pancreatic trypsin inhibitor } \\
\text { (BPTI) }\end{array}$ & Perioperative blood loss \\
\hline Arginine & Essential amino acid & Nutraceutical \\
\hline Aripiprazole & Atypical antipsychotic & $\begin{array}{l}\text { Bipolar disorder, major depressive } \\
\text { disorder, authistic disorder }\end{array}$ \\
\hline Artesunate & Antiprotozoal, antimalarian & Malaria \\
\hline Atenolol & $\begin{array}{l}\text { Competitive beta(1)-selective } \\
\text { adrenergic antagonist }\end{array}$ & Hypertension, angina pectoris \\
\hline Atorvastatin & $\begin{array}{l}\text { Selective, competitive HMG-CoA } \\
\text { reductase inhibitor (anti-cholesterol) }\end{array}$ & $\begin{array}{l}\text { Coronary heart disease, acute coronary } \\
\text { syndrome }\end{array}$ \\
\hline Atovaquone & $\begin{array}{l}\text { Synthetic hydroxynaphthoquinone } \\
\text { (antiprotozoal, antimalarian) }\end{array}$ & $\begin{array}{l}\text { Pneumocystis carinii pneumonia, } \\
\text { toxoplasmose }\end{array}$ \\
\hline $\begin{array}{l}\text { Atrial Natriuretic } \\
\text { Peptide }\end{array}$ & Polypeptide vasodilator & Heart failure \\
\hline Aspirin & NSAID & $\begin{array}{l}\text { Pain, swelling, reduces the risk of a } \\
\text { blood clot, prevent further heart } \\
\text { attacks or strokes }\end{array}$ \\
\hline Auranofin & Gold salt (anti-rheumatic) & RA \\
\hline Azithromycin & Macrolide antibiotic, semi-synthetic & Bacterial infection, CAP, PID \\
\hline
\end{tabular}




\begin{tabular}{|c|c|c|}
\hline Bazedoxifene & $\begin{array}{l}\text { Selective estrogen receptor } \\
\text { modulator }\end{array}$ & Osteoporosis \\
\hline Bedaquiline & $\begin{array}{l}\text { ATP-synthase inhibitor (antibiotic, } \\
\text { diaryl-quinoline ) }\end{array}$ & Tuberculosis \\
\hline Bemiparin & LMWH (anti-coagulant) & $\begin{array}{l}\text { Venous thromboembolism, myocardial } \\
\text { infarction }\end{array}$ \\
\hline Benserazide & $\begin{array}{l}\text { Peripherally-acting aromatic L-amino } \\
\text { acid decarboxylase (AADC) or DOPA } \\
\text { decarboxylase inhibitor }\end{array}$ & Parkinson's Disease \\
\hline Bepridil & Calcium Channel Blocker & Hypertension and chronic stable angina \\
\hline Bezafibrate & Fibrate & Hyperlidipemia \\
\hline Biperiden & Muscarinic antagonist & Parkinson's Disease \\
\hline Bosentan & Endothelin receptor antagonists & $\mathrm{PAH}$ \\
\hline Bromocriptine & Dopamine agonist & $\begin{array}{l}\text { Parkinson's Disease, prevention of } \\
\text { lactation }\end{array}$ \\
\hline Cabergoline & Dopamine receptor agonist & Hyperprolactinemia \\
\hline Caffeine & CNS Stimulant & Newborn apnea \\
\hline Calcitriol & Vitamin D3 & Vitamin \\
\hline Candesartan & Angiotensin Receptor Blocker & Hypertension \\
\hline Captopril & $\begin{array}{l}\text { Angiotensin-converting-enzyme } \\
\text { inhibitor }\end{array}$ & Anti-hypertension \\
\hline Carbimazole & Imidazole-derative (antithyroid) & Hyperthyroidism \\
\hline Carglumic acid & Metabolic alkalosis agent & $\begin{array}{l}\text { Hyperammonaemia in } \mathrm{N}- \\
\text { acetylglutamate synthase deficiency }\end{array}$ \\
\hline Carvedilol & Betablocker & Hypertension \\
\hline Celecoxib & NSAID (COX-2) & $\begin{array}{l}\text { OA, RA, JRA, AS, acute pain, primary } \\
\text { dysmenorrhea }\end{array}$ \\
\hline Cephalexin & Cephalosporin antibiotic & Bacterial infections \\
\hline Cholecalciferol & Vitamin D3 & Vitamin \\
\hline Chlorpromazine & Phenothiazine (typical antipsychotic) & $\begin{array}{l}\text { Psychotic disorders, nausea and } \\
\text { vomiting, anxiety, hiccups }\end{array}$ \\
\hline Chloroquine & Antimalarial and amebicidal drug & Malaria, Extraintestinal Amebiasis \\
\hline Cidofovir & $\begin{array}{l}\text { Deoxycytidine monophosphate } \\
\text { analog }\end{array}$ & CMV-retinitis in AIDS \\
\hline Cilnidipine & Calcium Channel Blocker & Hypertension \\
\hline Cimetidine & Histamine H2-receptor blocker & $\begin{array}{l}\text { Duodenal/gastric ulcers, GERD, } \\
\text { pathological hypersecretory conditions }\end{array}$ \\
\hline Ciprofloxacine & Fluoroquinolone & Antibiotic \\
\hline Citalopram & $\begin{array}{l}\text { Selective Serotonin Reuptake } \\
\text { Inhibitors }\end{array}$ & Depression \\
\hline Clarithromycin & Macrolide antibiotic & Bacterial infections \\
\hline Clofoctol & Other antibiotic & Bacterial infections \\
\hline Clomifene & Synthetic ovulation stimulant & Ovulatory dysfunction \\
\hline Clomipramine & Tricyclic anti-depressant & Obsessive Compulsive Disorder \\
\hline
\end{tabular}




\begin{tabular}{|c|c|c|}
\hline Clotrimazole & Imidazole derivative & Fungal infections \\
\hline Colchicine & Antimitotic alkaloid & Gout \\
\hline Dalteparin & LMWH (anti-coagulant) & $\begin{array}{l}\text { DVT (profylaxis), unstable angina/non- } \\
\text { Q-wave myocardial infarction }\end{array}$ \\
\hline Danazol & Antigonadotropins and similar agents & $\begin{array}{l}\text { Endometriosis, fibrocystic breast } \\
\text { disease, hereditary angioedema }\end{array}$ \\
\hline Dapsone & Antibiotic & Dermatitis herpetiformis, leprosy \\
\hline Deferoxamine & Iron chelating agent & $\begin{array}{l}\text { Acute iron intoxication, chronic iron } \\
\text { overload }\end{array}$ \\
\hline Desmopressin & Vasopressin analogue & $\begin{array}{l}\text { Diabetes Insipidus, } \\
\text { bedwetting, hemophilia A, von } \\
\text { Willebrand's disease }\end{array}$ \\
\hline Diclofenac & NSAID & OA, RA, AS \\
\hline Diflunisal & NSAID & OA, RA, mild to moderate pain \\
\hline Digitoxin & Cardiac glycoside & $\begin{array}{l}\text { Congestive } \mathrm{HF} \text {, atrial fibrillation, atrial } \\
\text { flutter, PAT, cardiogenic shock }\end{array}$ \\
\hline Digoxin & Cardiac glycoside & Heart failure, atrial fibrillation \\
\hline Dimethyl Fumarate & NA & Psoriasis, Multiple Sclerosis \\
\hline Dipyridamole & Platelet aggregation inhibitor & $\begin{array}{l}\text { Thromboembolism Prophylaxis Post- } \\
\text { Cardiac Valve Replacement }\end{array}$ \\
\hline Disulfiram & Alcohol antagonist & Chronic alcoholism \\
\hline Donepezil & Acetylcholinesterase inhibitor & Alzheimer's Disease \\
\hline Doxazosin & & Anti-hypertensive \\
\hline Doxycycline & & Antibiotic \\
\hline Ebastine & Histamine H1-receptor blocker & Allergies \\
\hline Efavirenz & & Anti-retroviral \\
\hline \multicolumn{3}{|l|}{ Eflornithine } \\
\hline Enalapril & $\begin{array}{l}\text { Angiotensin-converting-enzyme } \\
\text { inhibitor }\end{array}$ & Anti-hypertensive \\
\hline Enoxaparin & LMWH & Anti-coagulant \\
\hline Epalrestat & Aldose reductase inhibitor & Anti-diabetic \\
\hline Esomeprazole & Proton Pump Inhibitor & Antacid \\
\hline Ethacrynic acid & Loop diuretic & Diuretic \\
\hline Etodolac & NSAID (COX-2) & NSAID \\
\hline Famotidine & Histamine H2-receptor blocker & Antacid \\
\hline Fasudil & & Vasodilator \\
\hline Felodipine & Calcium Channel Blocker & Anti-hypertensive \\
\hline Fenofibrate & Fibrate & Anti-cholesterol \\
\hline Fingolimob & $\begin{array}{l}\text { Sphingosine-1-phosphate receptor } \\
\text { modulator }\end{array}$ & Multiple Sclerosis \\
\hline Fish oil (EPA/DHA) & & Anti-cholesterol \\
\hline Flubendazole & & Anti-parasitic \\
\hline Fluoxetine & & Anti-depressant \\
\hline
\end{tabular}




\begin{tabular}{|c|c|c|}
\hline Fluspirilene & & Anti-psychotic \\
\hline Fluvastatin & & Anti-cholesterol \\
\hline Fluvoxamine & $\begin{array}{l}\text { Serotonin antagonist and reuptake } \\
\text { inhibitors }\end{array}$ & Anti-depressant \\
\hline Glipizide & Sulfonylureas & Anti-diabetic \\
\hline Glutamine & & Nutraceutical \\
\hline Griseofulvin & & Anti-fungal \\
\hline Haloperidol & & Dopamine antagonist \\
\hline Hydralazine & & Anti-hypertensive \\
\hline \multicolumn{3}{|l|}{ Hydroxychloroquine } \\
\hline Hymecromone & & Antispasmodic \\
\hline Ibandronate & & Bisphosphonate \\
\hline Ibuprofen & & Analgesic \\
\hline Imipramine & Tricyclic Anti-depressant & Anti-depressant \\
\hline \multicolumn{3}{|l|}{ Imiquimod } \\
\hline Indomethacin & & NSAID \\
\hline Irbesartan & Angiotensin Receptor Blocker & Anti-hypertensive \\
\hline Itraconazole & & Anti-fungal \\
\hline Ivermectin & & Anti-parasitic \\
\hline Ketoconazole & & Anti-fungal \\
\hline Ketorolac & & NSAID \\
\hline Lanreotide & Somatostatin analogue & \\
\hline Lansoprazole & Proton Pump Inhibitor & Antacid \\
\hline Leflunomide & & Arthritis \\
\hline Levetiracetam & & Anti-epileptic \\
\hline Levofloxacin & Fluoroquinolone & Antibiotic \\
\hline Licofelone & & Osteoarthritis \\
\hline Lithium & & Bipolar disorders \\
\hline Lidocaine & & Anesthetic \\
\hline Loperamide & & Anti-diarrhea \\
\hline Loratadine & Histamine H1-receptor blocker & Allergies \\
\hline Losartan & Angiotensin Receptor Blocker & Anti-hypertensive \\
\hline Lovastatin & Statin & Anti-cholesterol \\
\hline Loxoprofen & NSAID & Anti-inflammatory \\
\hline Macitentan & Endothelin receptor antagonists & Pulmonary arterial hypertension \\
\hline Manidipine & Calcium Channel Blocker & Anti-hypertensive \\
\hline Maraviroc & CCR5 receptor antagonist & Anti-retroviral \\
\hline Mebendazole & & Anti-parasitic \\
\hline \multicolumn{3}{|l|}{ Meclofenamate } \\
\hline Megestrol acetate & & Hormone \\
\hline Mefloquine & & Anti-malarial \\
\hline Melatonin & & Anti-insomnia \\
\hline
\end{tabular}




\begin{tabular}{|c|c|c|}
\hline Meloxicam & NSAID & Anti-inflammatory \\
\hline Memantine & NMDA receptor antagonist & Alzheimer's Disease \\
\hline Mepacrine (Quinacrine) & & Anti-parasitic \\
\hline Metformin & & Anti-diabetic \\
\hline \multicolumn{3}{|l|}{ Methimazole } \\
\hline Methazolamide & & Antiglaucoma, diuretic \\
\hline Methylnaltrexone & $\mu$-opioid antagonist & Opioid-induced constipation \\
\hline Metoclopramide & & Anti-emesis \\
\hline Mifepristone & & Abortifacient \\
\hline Minocycline & & Antibiotic \\
\hline \multicolumn{3}{|l|}{ Mirtazapine } \\
\hline \multicolumn{3}{|l|}{ Montelukast } \\
\hline Mycophenolate & & Immunosuppressant \\
\hline \multicolumn{3}{|l|}{ Nadroparin } \\
\hline Naproxen & & NSAID \\
\hline Naltrexone & & Opioid receptor antagonist \\
\hline Nelfinavir & & Anti-retroviral \\
\hline Niclosamide & & Anti-parasitic \\
\hline Nifedipine & Calcium Channel Blocker & Anti-hypertensive \\
\hline \multicolumn{3}{|l|}{ Nifurtimox } \\
\hline Nimodipine & Calcium Channel Blocker & Anti-hypertensive \\
\hline Nisodilpine & Calcium Channel Blocker & Anti-hypertensive \\
\hline Nitazoxanide & & Anti-protozoal \\
\hline \multicolumn{3}{|l|}{ Nitisinone } \\
\hline Nitroxoline & & Antibiotic \\
\hline Nitroglycerine & & Nitro-vasodilator \\
\hline Norethandrolone & Androgen & Aplastic anemia \\
\hline Noscapine & & Anti-tussive \\
\hline \multicolumn{3}{|l|}{ Octreotide } \\
\hline Olanzapine & Atypical antipsychotic & Anti-psychotic \\
\hline Olsalazine & & Anti-inflammatory \\
\hline Omeprazole & Proton Pump Inhibitor & Antacid \\
\hline Orlistat & Lipase Inhibitor & Obesity \\
\hline Ormeloxifene & $\begin{array}{l}\text { Selective estrogen receptor } \\
\text { modulator }\end{array}$ & Contraceptive \\
\hline Oseltamivir & Neuraminidase inhibitor & Anti-viral \\
\hline Ouabain & & Anti-aryhtmic \\
\hline Oxcarbazepine & $\begin{array}{l}\text { Voltage-gated sodium channel } \\
\text { blocker }\end{array}$ & Epilepsy \\
\hline Pantoprazole & Proton Pump Inhibitor & Antacid \\
\hline Penfluridol & Diphenylbutylpiperidine & Anti-psychotic \\
\hline Pentamidine & & Anti-parasitic \\
\hline
\end{tabular}




\begin{tabular}{|c|c|c|}
\hline Pentoxifylline & Xanthine derivative & Peripheral artery disease \\
\hline Perphenazine & Phenothiazine & Anti-psychotic \\
\hline Phenylbutyrate & & Urea cycle disorders \\
\hline Phentolamine & Alfa-adrenergic antagonist & Vasodilator \\
\hline Phenytoin & & Anti-epileptic \\
\hline Pirfenidone & & Anti-fibrotic \\
\hline Pimozide & Diphenylbutylpiperidine & Anti-psychotic \\
\hline Pioglitazone & & Anti-diabetic \\
\hline Plerixafor & & Autologous HSCT \\
\hline Pravastatin & Statin & Anti-cholesterol \\
\hline Prazosin & & Anti-hypertensive \\
\hline Pregabalin & Gabapentinoid & Anti-convulsant \\
\hline Promethazine & Phenothiazine & Anti-psychotic \\
\hline Propranolol & Betablocker & Anti-hypertensive \\
\hline Pyrimethamine & Dihydrofolate reductase inhibitor & Anti-parasitic \\
\hline Pyrvinium pamoate & & Anti-parasitic \\
\hline Quetiapine & Atypical antipsychotic & Anti-psychotic \\
\hline Rabeprazole & Proton Pump Inhibitor & Antacid \\
\hline Ranolazine & $\begin{array}{l}\text { Voltage-gated sodium channel } \\
\text { blocker }\end{array}$ & Anti-angina \\
\hline Repaglinide & & Anti-diabetic \\
\hline Ribavirin & nucleoside inhibitor & Anti-viral \\
\hline Rifabutin & & Antibiotic \\
\hline Riluzole & & ALS \\
\hline Risperidone & Atypical antipsychotic & Anti-psychotic \\
\hline Ritonavir & Protease inhibitor & Anti-HIV \\
\hline Roflumilast & PDE-4 inhibitor & COPD \\
\hline Rosuvastatin & Statin & Anti-cholesterol \\
\hline Sertraline & & Anti-depressant \\
\hline Sildenafil & PDE-5 inhibitor & Erectile dysfunction \\
\hline Simvastatin & Statin & Anti-cholesterol \\
\hline Sirolimus & & Inhibit organ transplant rejection \\
\hline Sodium Bicarbonate & & Antacid \\
\hline \multicolumn{2}{|l|}{ Sodium Oxybate } & Narcolepsy \\
\hline Spironolactone & & Anti-hypertensive \\
\hline Sulfasalazine & & Anti-rheumatic \\
\hline Sulindac & & NSAID \\
\hline Tadalafil & PDE-5 inhibitor & Erectile dysfunction \\
\hline Terbinafine & & Anti-fungal \\
\hline Telmisartan & Angiotensin Receptor Blocker & Anti-hypertensive \\
\hline Tetrathiomolybdate & & Copper toxicosis \\
\hline Thalidomide & Immunomodulatory imide drug & Leprosy, multiple myeloma \\
\hline
\end{tabular}




\begin{tabular}{|l|l|l|}
\hline Thiabendazole & & Anti-parasitic \\
\hline Thioridazine & Penothiazine & Anti-psychotic \\
\hline Ticagrelor & & Anti-platelet \\
\hline Ticlopidine & Glycylcycline & Anti-platelet \\
\hline Tigecycline & Betablocker & Antibiotic \\
\hline Timolol & LMWH & Anti-hypertensive \\
\hline Tinzaparin & Fenamate NSAIDs & Anti-coagulant \\
\hline Tolfenamic acid & & NSAID \\
\hline Topiramate & & Epilepsy \\
\hline Tranexamic acid & Serotonin antagonist and reuptake & Anti-depressant \\
\hline Trazodone & inhibitors & \\
\hline Triamterene & Potassium-sparing diuretic & Diuretic \\
\hline Trifluoperazine & Phenothiazine & Anti-psychotic \\
\hline Ulinastatin & Trypsine inhibitor & Severe sepsis \& pancreatitis \\
\hline Urokinase & & \\
\hline Valproic acid & & Anti-convulsant \\
\hline Valsartan & Angiotensin Receptor Blocker & Anti-hypertensive \\
\hline Verapamil & & Anti-hypertensive \\
\hline Warfarin & Anti-Vitamin-K & Anti-coagulant \\
\hline Zoledronate & & Bisphosphonate \\
\hline & & \\
\hline
\end{tabular}

Table 2: Some features of the $\mathbf{2 3 5}$ drugs listed

\begin{tabular}{|l|l|l|}
\hline & $\mathbf{N}$ & $\%$ \\
\hline Human data (at least 1 case report, 1 obs. study or 1 clinical trial) & 133 & $\mathbf{5 7 \%}$ \\
\hline At least 1 clinical trial & 124 & $\mathbf{5 3 \%}$ \\
\hline Drug Off-Patent & 176 & $\mathbf{7 5 \%}$ \\
\hline
\end{tabular}

\section{Discussion}

We have found that the number of off-patent repurposing opportunities is large and increasing.

Until now, practice-changing examples have been limited despite the evidence. Joint non-commercial clinical development (academics, governments, charities) may bring new therapeutic options to patients at low cost, especially in indications for which the industry has no incentive to invest in. This may relieve healthcare systems currently under high financial stress.

A change in market authorisation regulation will be required to avoid off-label use. 
The Anticancer Fund is actively pursuing this innovative strategy, as exemplified by the granting of an EMA orphan designation for propranolol in angiosarcoma.

\section{References}

1. Pantziarka P, Bouche G, Meheus L, Sukhatme V, Sukhatme VP, Vikas P. The Repurposing Drugs in Oncology (ReDO) Project. Ecancermedicalscience. 2014;8:442. doi:10.3332/ecancer.2014.442.

2. Bertolini F, Sukhatme VP, Bouche G. Drug repurposing in oncology-patient and health systems opportunities. Nat Rev Clin Oncol. October 2015:1-11. doi:10.1038/nrclinonc.2015.169.

3. Califano J a., Khan Z, Noonan K a., et al. Tadalafil augments tumor specific immunity in patients with head and neck squamous cell carcinoma. Clin Cancer Res. 2015;21(1):30-38. doi:10.1158/1078-0432.CCR-14-1716.

4. Stiles JM, Amaya C, Rains S, et al. Targeting of beta adrenergic receptors results in therapeutic efficacy against models of hemangioendothelioma and angiosarcoma. PLoS One. 2013;8(3):e60021. doi:10.1371/journal.pone.0060021.

5. Chow W, Amaya CN, Rains S, Chow M, Dickerson EB, Bryan B a. Growth Attenuation of Cutaneous Angiosarcoma With Propranolol-Mediated $\beta$-Blockade. JAMA Dermatology. 2015;(June 2014):1. doi:10.1001/jamadermatol.2015.2554.

6. Chisholm KM, Chang KW, Truong MT, Kwok S, West RB, Heerema-McKenney AE. $\beta$-Adrenergic receptor expression in vascular tumors. Mod Pathol. 2012;25(11):1446-1451. doi:10.1038/modpathol.2012.108.

7. Mestres J, Gregori-Puigjané E, Valverde S, Solé R V. Data completeness--the Achilles heel of drugtarget networks. Nat Biotechnol. 2008;26(9):983-984. doi:10.1038/nbt0908-983.

8. Joly $\mathrm{P}$, Maho-Vaillant $\mathrm{M}$, Prost-Squarcioni $\mathrm{C}$, et al. First-line rituximab combined with short-term prednisone versus prednisone alone for the treatment of pemphigus (Ritux 3): a prospective, multicentre, parallel-group, open-label randomised trial. Lancet (London, England). 2017;(Ritux 3):2031-2040. doi:10.1016/S0140-6736(17)30070-3.

9. Fornoni A, Sageshima J, Wei C, et al. Rituximab targets podocytes in recurrent focal segmental glomerulosclerosis. Sci Transl Med. 2011;3(85):85ra46. doi:10.1126/scitranslmed.3002231.

10. Bogdanovich S, Kim Y, Mizutani T, et al. Human IgG1 antibodies suppress angiogenesis in a targetindependent manner. Signal Transduct Target Ther. 2015;1(August 2015):1-18. doi:10.1038/sigtrans.2015.1.

11. Verbaanderd C, Meheus L, Huys I, Pantziarka P. Repurposing Drugs in Oncology: Next Steps. Trends in cancer. 2017;3(8):543-546. doi:10.1016/j.trecan.2017.06.007.

12. Sukhatme VP, Fang K, Lo A, Sukhatme V. Financial Orphan Therapies Looking For Adoption. Health Affairs Blog. http://healthaffairs.org/blog/2014/03/06/financial-orphan-therapies-lookingfor-adoption/. Published 2014.

13. Deva S, Jameson M. Histamine type 2 receptor antagonists as adjuvant treatment for resected colorectal cancer. Cochrane database Syst Rev. 2012;8(8):CD007814. doi:10.1002/14651858.CD007814.pub2.

14. Badwe R, Hawaldar R, Parmar V, et al. Single-injection depot progesterone before surgery and survival in women with operable breast cancer: a randomized controlled trial. J Clin Oncol. 2011;29(21):2845-2851. doi:10.1200/JCO.2010.33.0738.

15. Rudin CM, Brahmer JR, Juergens RA, et al. Phase 2 study of pemetrexed and itraconazole as second-line therapy for metastatic nonsquamous non-small-cell lung cancer. J Thorac Oncol. 2013;8(5):619-623. doi:10.1097/JTO.0b013e31828c3950. 
16. Ohe M, Hashino S. A case of follicular B-cell lymphoma treated using clarithromycin. Korean J Hematol. 2011;46(3):203-206. doi:10.5045/kjh.2011.46.3.203.

17. Alcántara-Reifs CM, Salido-Vallejo R, Garnacho-Saucedo GM, Vélez García-Nieto A. Classic Kaposi's sarcoma treated with topical 0.5\% timolol gel. Dermatol Ther. 2016;29(5):309-311. doi:10.1111/dth.12381.

18. Meseguer-Yebra C, Cardeñoso-Álvarez ME, Bordel-Gómez MT, Fraile-Alonso MC, Pérez-Losada ME, Sánchez-Estella J. Successful treatment of classic Kaposi sarcoma with topical timolol: report of two cases. Br J Dermatol. 2015;173(3):860-862. doi:10.1111/bjd.13746.

19. Chow W, Amaya CN, Rains S, Chow M, Dickerson EB, Bryan BA. Growth Attenuation of Cutaneous Angiosarcoma With Propranolol-Mediated $\beta$-Blockade. JAMA dermatology. 2015;151(11):12261229. doi:10.1001/jamadermatol.2015.2554.

20. Daguzé J, Saint-Jean M, Dréno B. Large nose angiosarcoma treated effectively with oral cyclophosphamide combined with propranolol. J Eur Acad Dermatology Venereol. 2017;2. doi:10.1111/jdv.14528.

21. Daguzé J, Saint-Jean $M$, Peuvrel L, et al. Visceral metastatic angiosarcoma treated effectively with oral cyclophosphamide combined with propranolol. JAAD Case Reports. 2016;2(6):497-499. doi:10.1016/j.jdcr.2016.10.005.

22. Pasquier E, André N, Street J, et al. Effective Management of Advanced Angiosarcoma by the Synergistic Combination of Propranolol and Vinblastine-based Metronomic Chemotherapy: A Bench to Bedside Study. EBioMedicine. 2016;6:87-95. doi:10.1016/j.ebiom.2016.02.026. 